\title{
Glaucomatous Macular Vasculature: A Quantitative Analysis
}

\author{
Pedro Arede ${ }^{1}$, Joanna Przezdziecka-Dolyk ${ }^{2,3}$, Fabian Debowy ${ }^{2}$, Jacek Olszewski ${ }^{2}$, Carla Fernandes ${ }^{1}$, Carolina Bruxelas ${ }^{1}$, Helena \\ Spohr ${ }^{1}$, Sara Patricio ${ }^{1}$ \\ ${ }^{1}$ Ophthalmology Department of Egas Moniz Hospital, Centro Hospitalar Lisboa Ocidental, Lisbon, Portugal \\ ${ }^{2}$ Department of Optics and Photonics, Wrocław University of Science and Technology, Poland \\ ${ }^{3}$ Department of Ophthalmology, Medical University of Wrocław, Poland
}

\begin{abstract}
Background: The aim of this study was to evaluate the characteristics of the macular vessel density (VD) and the foveal avascular zone (FAZ) in glaucoma quantitatively using the optical coherence tomography angiography (OCT-A). Methods: Twenty-five eyes of 13 patients with primary open angle glaucoma (POAG) and 12 eyes of 6 healthy participants were enrolled retrospectively. Functional visual field (VF) and structural Spectral-Domain optical coherence tomography (SD-OCT) Retinal Nerve Fiber Layer Thickness (RNFLT) were assessed in all participants. OCT-A was performed on a fovea centered, $15 \times 10$ degrees, macular region. OCT-A scans were processed with MATLAB software and automatically graded to define FAZ parameters. The parafoveal VD in the superficial and deep retinal vascular plexus (SVP and DVP) was analyzed by quadrant and circular segmented zones.

Results: Foveal Avascular Zone -Major Axis Length $(p=0.02)$, Area $(p=0.04)$, Equivalent Diameter $(p=0.04)$ and Perimeter $(p=0.04)$ were significantly larger in glaucoma than the control group. Regarding SVP and DVP, the average macular total VD were lower in glaucoma patients compared to the control group $(p<0.01 ; p<0.01)$. Additionally, the inner circular region $(p=0.04 ; p<0.01$ respectively for SVP and DVP) and all quadrants except for North had a lower VD in glaucoma group compared to the control group. Assessment of the total VD successfully predicted RNFLT $(p<0.001)$ and was significantly associated with the probability of glaucoma $(p=0.009)$.

Conclusions: OCT-A parameters, namely the FAZ morphology and the macular VD, were associated with glaucomatous functional and structural changes. The macular VD showed a considerable diagnostic value. It may be a modern biomarker, representing microvascular network disruption of the macular perfusion in glaucoma.
\end{abstract}

\section{KEY WORDS}

Glaucoma; OCT-A; Vascular Dropout; Macular Vessel Density; Foveal Avascular Zone; Biometry; Optical Coherence Tomography Angiography; Biomarker; Macular Perfusion; Superficial Vascular Plexus; Deep Vascular Plexus

Copyright (C 2020, Med Hypothesis Discov Innov Ophthalmol. This is an open-access article distributed under the terms of the Creative Commons Attribution-NonCommercial 4.0 International License (http://creativecommons.org/licenses/by-nc/4.0/) which permits copy and redistribute the material just in noncommercial usages, provided the original work is properly cited.

Correspondence to: Pedro Arede MD; Ophthalmology Department of Egas Moniz Hospital, Piso 2, R. da Junqueira 126, 1349-019 Lisboa, Portugal. E-mail: pedrovidalarede@gmail.com

How to cite this article: Arede P, Przezdziecka-Dolyk J, Debowy F, Olszewski J, Fernandes C, Bruxelas C, Spohr H, Patricio H, Glaucomatous Macular Vasculature; A Quantitative Analysis. Med Hypothesis Discov Innov Ophthalmol. 2020; 9(3): 185-197.

\section{INTRODUCTION}

As one of the world's leading causes of blindness, glaucoma is a neurodegenerative disorder characterized by selective retinal ganglion cell (RGC) loss with corresponding optic nerve head (ONH) cupping and VF defects [1]. The two leading theories of pathophysiological changes in the course of glaucoma include dysregulation of the apoptotic process that affects the RGCs and microvascular abnormalities that with increased 
intraocular pressure (IOP) cause hypoxia and reperfusion injury to the RGCs and their axons. Furthermore, other mechanisms including genetic factors (susceptibility genes), prolonged steroid use and autoimmune processes has been described, previously [2].

The biomechanical stress caused by IOP is considered as the most important modifiable risk factor. In previous studies, short-term changes of IOP have been defined as those bearing the potentially harmful factor to the RGC [3-5]. The increase of IOP after water-upload that exceeds $5 \mathrm{mmHg}$ from baseline was associated with 6 fold higher risk of new development of glaucoma [3]. Additionally, a difference higher than $1.9 \mathrm{mmHg}$ in IOP peak after waterupload was found in eyes with progressing glaucoma compared to those with a stable disease [5]. Taking into account the impact of IOP on the retinal arteries, that supply the RGC via superficial and deep plexus, the hypoxia-reperfusion injury is the most interesting field of research. The pre-perimetrical glaucoma and its probable progression to the new onset glaucoma needs intensive research. Additionally, it is not clear whether biomechanical stress equally affects retinal arteries and veins due to differences in their wall resistance factor.

The optical coherence tomography angiography (OCT-A) is a new and non-invasive retinal microvasculature imaging device, which has become available to assess vascular integrity [6, 7]. OCT-A provides high resolution and reproducible images of the macular microvascular network [8]. Unfortunately, the distinction between retinal veins and arteries cannot be ensured by means of OCT-A images. Another drawback of OCT-A is the arbitrarily pre-defined border between signal and noise. If particles in the retina move too slow or too fast over the set time frame, they would be considered as noise and removed from the analysis [9].

In previous studies, there was a significant association between Vessel Density (VD) of the ONH and structural glaucomatous damage [10-12] and that the peripapillary
VD measurements were comparable to the retinal nerve fiber layer measurement in terms of diagnostic accuracy [13]. The vascular supply of the $\mathrm{ONH}$ is complex. The prelaminar part is supplied only by the retinal vessels, but the laminar and retrolaminar parts are supplied by the combined retinal and ciliary vessels. In Zinn-Haller annulus, retinal and ciliary vessels create anastomoses [14].

Nevertheless, little is still known about vascular changes in the macular region in pre-perimetric and perimetric glaucoma. In contrast to the optic nerve region, in the macular region there is no circulation overlap as the neural retina is supplied only by the retinal vascular system and the outer retina is supplied by the choroidal system. The clinical meaning of the microcirculation status in glaucoma is still not completely understood [15].

The purpose of this study was to investigate the characteristics of the macular vascular architecture in eyes with POAG.

\section{METHODS}

A retrospective case-control investigation was performed based on the medical database from an Ophthalmology Department of a tertiary hospital (Hospital Egas Moniz, Lisboa) from June 2018 to September 2018 and included all patients examined in this center. This study was conducted as a part of the study registered as https://clinicaltrials.gov/ Identifier: NCT03928665, controls group (glaucoma and healthy controls).

Twenty-five eyes of 13 patients with POAG and 12 eyes of 6 healthy participants were enrolled. During the screening, patients were preselected according to the inclusion criteria (Table 1). If the patient had a unilateral glaucomatous change, the affected eye was selected, but if both eyes were affected, both were selected for entry. Bilateral healthy participants' eyes were included (Table 1, Control Group).

Table 1. Inclusion and Exclusion Criteria

\begin{tabular}{|c|c|}
\hline Inclusion C & Exclusion Criteria \\
\hline $\begin{array}{l}\text { Glaucoma Group: } \\
\text { - Glaucomatous ONH cup on stereoscopic fundoscopy, associated with } \\
\text { structural OCT Spectral-Domain (Heidelberg Engineering®, Heidelberg, } \\
\text { Germany)-RNFL damage. } \\
\text { - Functional VF defect by standard automated perimetry (Octopus 101, } \\
\text { Haag Streit }{ }^{\circledR}, \text { The USA). } \\
\text { Open angle on gonioscopic examination. } \\
\text { Control Group: } \\
\text { - IOP less than } 21 \mathrm{mmHg} \text { with no history of elevated IOP. } \\
\text { - Normal appearance of ONH, undamaged neuroretinal rim, and RNFLT/ } \\
\text { - Normal VF. }\end{array}$ & $\begin{array}{l}\text { - Severe myopic fundus changes and ONH tilt that } \\
\text { impaired adequate ONH/VF evaluation. } \\
\text { - } \quad \text { Retinal or neurologic diseases that impact the VF. } \\
\text { - } \quad \text { Poor quality imaging scans with significant artifacts. } \\
\text { - History of diabetes mellitus or retinal vascular diseases } \\
\text { that can affect FAZ microvasculature. } \\
\text { Patients with obstructive sleep apnea. }\end{array}$ \\
\hline
\end{tabular}

Abbreviation: ONH: Optic Nerve head; OCT: Optical Coherence Tomography, RNFL: Retinal Nerve Fiber Layer, IOP: Intraocular Pressure, RNFLT: Retinal Nerve Fiber Layer Thickness, VF: Visual Field, FAZ: Foveal Avascular Zone. mmHg: millimetre of mercury. 
All patients underwent a complete ophthalmic and optometric examination, with medical history interview, measurement of best-corrected visual acuity (BCVA), slitlamp biomicroscopy, the Shaffer's gonioscopic angle classification [16], the Goldmann applanation tonometry and the optic disc and retinal examination (dilated stereoscopic examination). All subjects were submitted to standard automated perimetry, SD-OCT peripapillary Retinal Nerve Fiber Layer Thickness (RNFLT) measurement and OCT-A (Heidelberg Engineering, Heidelberg, Germany) examination.

The OCT-A scans were centered on the fovea and the builtin OCT software was applied to extract layers of superficial and deep retinal capillary plexus from the recorded structural OCT data. Individual scans in acquired series differed slightly in contrast. Therefore as a preprocessing step we performed Contrast Limited Adaptive Histogram Equalization (CLAHE) $[17,18]$ for each image, using a builtin MATLAB (The MathWorks, Inc., Natick, MA) routine with default parameters set. Each image has an angular field of view of $15 \times 10$ degrees, corresponding to the size of $1408 \times 917$ pixels. Additionally, a pixel aspect ratio of 1.0 was set, resulting in an image scale equal to 325 pixels per $\mathrm{mm}$.
The macular VD was assessed using MATLAB image processing algorithm, as previously described [15]. Each superficial and deep retinal vessel macular layer image (Fig. 1 a) was analyzed. The images were processed assuming that levels of signal intensity were directly proportional to the intravascular flow rate. The flow rate increased with the cross-section area of the capillary. Therefore, we segmented retinal images using the k-means method, assigning pixels' intensities into three clusters interpreted as a background intensity set, small vessels intensity set and large vessels intensity set, as shown in Figure 1.

Simultaneously, using low-pass morphological filtering and the active contour method, we found the FAZ. We identified FAZ perimeter pixels and the FAZ center. The FAZ region was excluded in the further analysis. This assessment differs from the previously presented by Choi et al. in 2017 who assessed FAZ using ImageJ in a separate analysis. In contrast, in this study each image was processed simultaneously regarding vascular density and the FAZ [15].

In the next step, the results of the conducted analysis were presented according to previously described method by Choi et al. Figure 1 [15].
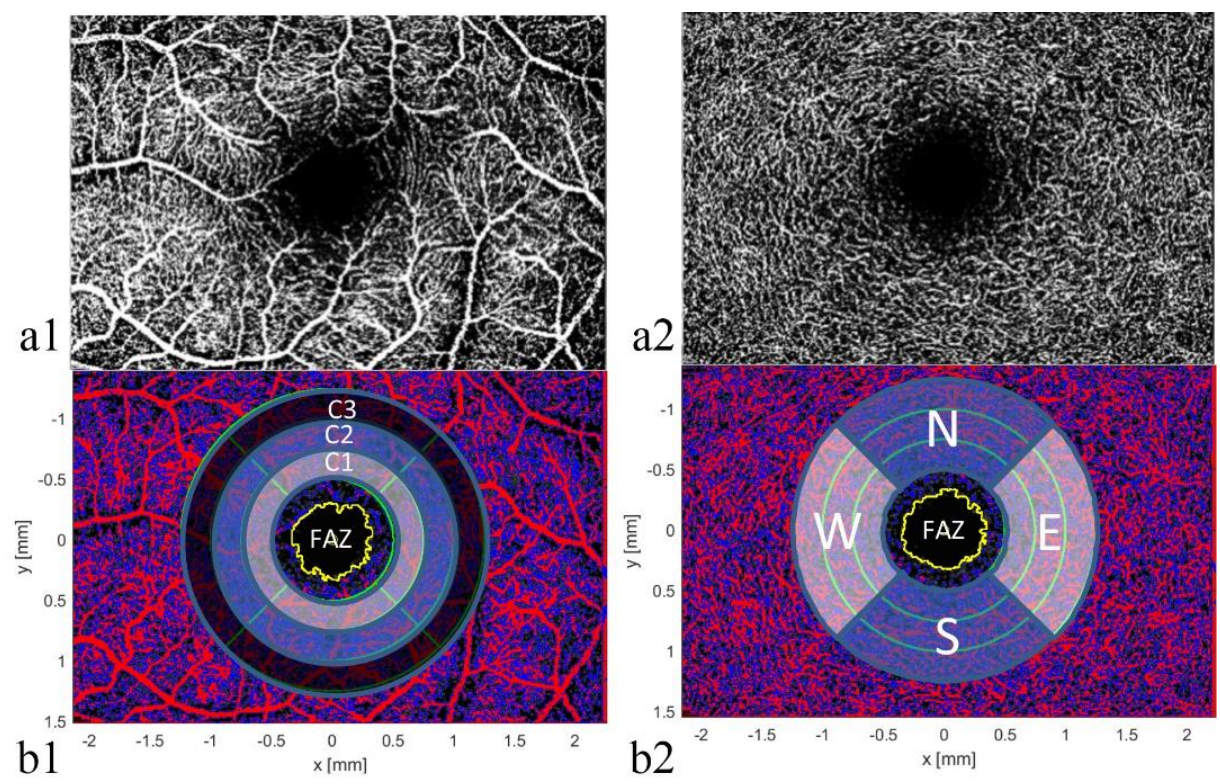

Figure 1. OCT-A Scan Images Processed With MATLAB Software (The MathWorks, Inc., Natick, MA); a) Left Eye, Healthy Control, 15x10 Degrees, Macular Scan of Superficial (a.1) and Deep (a.2) Capillary Plexus Before Image Enhancement, Algorithm Processing and Software Analysis; b) Automatically Generated Images of the Same Eye Defining Foveal Avascular Zone (FAZ) Area and Circular Segmented (C1: 1.0-1.5 mm; C2: 1.5-2.0 mm; C3: 2.0-2.5 mm) or Quadrant (N: North; S: South; E: East; W: West) Macular Zones of Superficial (b.1) and Deep (b.2) Capillary Plexus. Abbreviations: optical coherence tomography angiography (OCT-A) 
Another indicator used was the circularity index $(\mathrm{Cl})$, which has been already described by Choi et al.[15]. $\mathrm{Cl}$ is a measure of the similarity of a shape compared to that of a circle. FAZ circularity was included in the analysis due to decreasing $\mathrm{FAZ} \mathrm{Cl}$ when there is progressive disruption of the parafoveal capillary network.

Descriptive statistics for baseline demographics and clinical parameters associated with functional and structural glaucomatous damage were expressed as mean \pm standard deviation (SD), 95\% confidence intervals, and a median and interquartile range (IQR). The functional parameters included mean sensitivity (MS), mean deviation (MD) and Central VF Sensitivity (CVFS). The structural parameters included quadrant and average peripapillary RNFLT measured by OCT. To minimize the influence of potential bias arising from the inclusion of two eyes from one patient, we used Z-statistics and other statistical procedures. Categorical variables were compared using the sample-size-corrected chi-square (Vsquare) for non-parametric analyses. Differences in continuous variables between the groups were determined using Mann-Whitney $U$ test for independent samples. To evaluate the VD and FAZ-related parameters, the superficial and deep retinal vascular plexuses in each circular and quadrant segmentation were compared between patients with glaucoma and the control group. Associations between macular VD's, FAZ-related parameters, and structural and functional glaucomatous damage parameters were determined using a Spearman rank correlation test. After a preliminary assessment of existing correlations, the stepwise model builder was introduced. Firstly, the Macular VD and FAZ-related parameters were used to predict the glaucoma outcome. Multivariate linear regression models (built with the stepwise method) for the average RNFLT were separately built for the superficial and deep retinal vascular plexus (SVP and DVP) and FAZ-related parameters. Secondly, those same parameters were used in the stepwise logit probability model to determine the probability of having glaucoma. All tests were considered significant if $p$ value was below $5 \%$. The statistical analysis was performed using STATISTICA software 13.1 (StatSoft 1984-2016, Inc.).

\section{RESULTS}

Mean age \pm SD of patients was $68.1 \pm 10.33$ (median: 65 ) years. $72 \%(n=18)$ of the patients had glaucoma family history. The mean \pm SD IOP was $17.96 \pm 3.76 \mathrm{mmHg}$, using a mean of 2 anti-glaucomatous drugs. $90 \%$ of the cases were phakic with a mean BCVA of 0.72 (decimal scale, Snellen chart). All glaucomatous eyes had a gonioscopically open angle, Schaffer grade III $(56 \%, n=14)$ or grade IV $(44 \%, n=11)$. The control patients' mean age \pm SD was $36.7 \pm 6.9$ years. The mean \pm SD registered IOP was $16.9 \pm 3.5 \mathrm{mmHg}$. All controls were phakic with a mean BCVA of 1.0. Tables 2, 3 and 4 present demographic and clinical parameters for each group.

Table 2. Demographic and Clinical Parameters of Each Study Group

\begin{tabular}{|c|c|c|}
\hline & Glaucoma Group ( $n=25)$ & Control Group ( $n=12)$ \\
\hline \multicolumn{3}{|l|}{ DEMOGRAPHIC PARAMETERS } \\
\hline Age (mean $\pm S D)$ & $68.13 \pm 10.3$ & $36.7 \pm 6.9$ \\
\hline Gender; n (male:female) & 11:14 & $6: 6$ \\
\hline Glaucoma family history; $n$ (Yes: No) & $18: 7$ & $0: 6$ \\
\hline \multicolumn{3}{|l|}{ CLINICAL PARAMETERS } \\
\hline IOP; mean $\pm S D(m m H g)$ & $17.96 \pm 3.76$ & $16.9 \pm 3.5$ \\
\hline Number of anti-glaucomatous drugs; $n$ & 2 & 0 \\
\hline BCVA mean (decimal scale) & 0.72 & 1 \\
\hline Phakic status; n (Phakic:Pseudophakic) & $22: 25$ & $12: 0$ \\
\hline Pachymetry mean $(\mu \mathrm{m})$ & 555 & 570 \\
\hline Gonioscopic Shaffer Classification; n (III:IV) & $14: 11$ & $0: 12$ \\
\hline
\end{tabular}

Abbreviations: n: number; SD: standard deviation; IOP: intraocular pressure; mmHg: millimeter of mercury; BCVA: best corrected visual acuity; $\mu \mathrm{m}$ : micrometer.

Table 3. Functional Outcome, Automated Perimetry, and VF Parameters of Each Study Group

\begin{tabular}{|c|c|c|}
\hline & Glaucoma Group (n=25) & Control Group ( $n=12)$ \\
\hline MS (dB) (mean $\pm S D)$ & $18.8 \pm 8.65$ & $27.11 \pm 1.54$ \\
\hline $\mathrm{MD}(\mathrm{dB})($ mean $\pm S D)$ & $9.2 \pm 7.57$ & $1.2 \pm 0.7$ \\
\hline CVFS (dB) (mean $\pm S D)$ & $22.7 \pm 9.55$ & $35 \pm 3.1$ \\
\hline
\end{tabular}

Abbreviations: n: number; MS: Mean Sensitivity; MD: Mean Deviation; CVFS: Central Visual Field Sensitivity; dB: decibel; SD: Standard Deviation 
Table 4. Structural Outcome of OCT and Peripapillary RNFLT Parameter of Each Study Group

\begin{tabular}{|c|c|c|}
\hline & Glaucoma Group (n=23) & Control Group ( $\mathrm{n}=10)$ \\
\hline Total Average $(\mu \mathrm{m})($ mean $\pm S D)$ & $70.91 \pm 14.7$ & $101.10 \pm 6.2$ \\
\hline \multicolumn{3}{|l|}{ Quadrant measurements $(\mu \mathrm{m})$} \\
\hline Nasal Superior $(\mu \mathrm{m})($ mean $\pm S D)$ & $85.17 \pm 20.9$ & $115.40 \pm 16.0$ \\
\hline Temporal Superior $(\mu \mathrm{m})($ mean $\pm S D)$ & $100.52 \pm 25.3$ & $137.80 \pm 13.1$ \\
\hline Nasal Inferior $(\mu \mathrm{m})($ mean $\pm S D)$ & $81.56 \pm 26.2$ & $122.00 \pm 16.1$ \\
\hline Temporal Inferior $(\mu \mathrm{m})($ mean $\pm S D)$ & $87.08 \pm 33.2$ & $134.10 \pm 12.0$ \\
\hline Nasal $(\mu \mathrm{m})($ mean $\pm S D)$ & $54.60 \pm 15.0$ & $76.80 \pm 4.2$ \\
\hline Temporal $(\mu \mathrm{m})($ mean $\pm S D)$ & $52.78 \pm 10.0$ & $72.50 \pm 12.7$ \\
\hline
\end{tabular}

Abbreviations: SD: standard deviation; OCT: Optical coherence tomography; RNFLT: retinal nerve fiber layer thickness; $\mathrm{n}$ : number; $\mu \mathrm{m}$ : micrometer.

Table 5. Assessment of Macular Vessele Dencity Percentage by Optical Coherence Tomography Angiography in Each Study Group

\begin{tabular}{|c|c|c|c|}
\hline & Glaucoma Group ( $n=23$ ) & Control Group (n=12) & p-value \\
\hline \multicolumn{4}{|l|}{ OVERALL } \\
\hline SVP (mean $\pm S D)$ & $30.53 \pm 2.4$ & $33.15 \pm 0.2$ & $<0.0001$ \\
\hline DVP (mean $\pm S D)$ & $30.84 \pm 1.7$ & $33.18 \pm 0.1$ & $<0.0001$ \\
\hline \multicolumn{4}{|c|}{ QUADRANT SECTORS } \\
\hline \multicolumn{4}{|l|}{ North } \\
\hline SVP $($ mean $\pm S D)$ & $37.32 \pm 4.0$ & $36.48 \pm 1.2$ & 0.43 \\
\hline $\mathrm{DVP}($ mean $\pm S \mathrm{D})$ & $34.72 \pm 1.4$ & $35.28 \pm 0.6$ & 0.11 \\
\hline \multicolumn{4}{|l|}{ South } \\
\hline SVP $($ mean $\pm S D)$ & $34.28 \pm 7.3$ & $37.21 \pm 1.1$ & 0.09 \\
\hline DVP (mean $\pm S D)$ & $32.80 \pm 2.6$ & $35.64 \pm 0.5$ & $<0.0001$ \\
\hline \multicolumn{4}{|l|}{ East } \\
\hline SVP (mean $\pm S D)$ & $36.19 \pm 3.7$ & $37.78 \pm 0.7$ & 0.03 \\
\hline DVP $($ mean $\pm S D)$ & $32.43 \pm 2.0$ & $35.30 \pm 1.1$ & $<0.0001$ \\
\hline \multicolumn{4}{|l|}{ West } \\
\hline SVP $($ mean $\pm S D)$ & $33.83 \pm 4.6$ & $36.74 \pm 1.4$ & 0.03 \\
\hline DVP (mean $\pm S D)$ & $31.69 \pm 2.5$ & $34.41 \pm 1.1$ & 0.0003 \\
\hline \multicolumn{4}{|c|}{ CIRCULAR SECTORS (C) } \\
\hline \multicolumn{4}{|l|}{ C1 } \\
\hline SVP (mean $\pm S D)$ & $34.38 \pm 5.7$ & $37.92 \pm 1.4$ & 0.04 \\
\hline DVP (mean $\pm S D)$ & $30.90 \pm 3.1$ & $35.20 \pm 1.2$ & $<0.0001$ \\
\hline \multicolumn{4}{|l|}{ C2 } \\
\hline SVP $($ mean $\pm S D)$ & $36.75 \pm 4.0$ & $37.85 \pm 0.7$ & 0.41 \\
\hline DVP $($ mean $\pm S D)$ & $33.94 \pm 2.1$ & $35.67 \pm 1.0$ & 0.01 \\
\hline \multicolumn{4}{|l|}{ C3 } \\
\hline SVP (mean $\pm S D)$ & $34.94 \pm 2.9$ & $35.95 \pm 0.8$ & 0.37 \\
\hline DVP (mean $\pm S D)$ & $33.23 \pm 1.2$ & $34.73 \pm 0.4$ & 0.0009 \\
\hline
\end{tabular}

Abbreviations: n: number; SD: standard deviation; VD: Vessel Density; SVP: Superficial Vessel Plexus; DVP: Deep Vessel Plexus. P<0.05 in bold.

Table 6. Assessment of FAZ by Optical Coherence Tomography Angiography in Each Study Group

\begin{tabular}{|c|c|c|c|}
\hline & Glaucoma Group ( $n=23$ ) & Control Group ( $n=12$ ) & p-value \\
\hline FAZ area $\left(\mathrm{mm}^{2}\right)($ mean $\pm S D)$ & $0.33 \pm 0.13$ & $0.25 \pm 0.05$ & 0.04 \\
\hline FAZ Major Axis Length $(\mathrm{mm})$ (mean $\pm S D)$ & $0.69 \pm 0.17$ & $0.60 \pm 0.06$ & 0.02 \\
\hline FAZ Minor Axis Length $(\mathrm{mm})$ (mean $\pm S D)$ & $0.59 \pm 0.13$ & $0.54 \pm 0.06$ & 0.06 \\
\hline FAZ Equivalent Diameter (mm) (mean $\pm S D)$ & $0.63 \pm 0.14$ & $0.57 \pm 0.06$ & 0.04 \\
\hline FAZ Perimeter $(\mathrm{mm})($ mean $\pm S D)$ & $2.14 \pm 0.53$ & $1.87 \pm 0.21$ & 0.04 \\
\hline $\mathrm{FAZ} \mathrm{Cl}(\mathrm{mm})($ mean $\pm \mathrm{SD})$ & $0.88 \pm 0.05$ & $0.91 \pm 0.03$ & 0.95 \\
\hline
\end{tabular}

Abbreviations: $\mathrm{n}$ : number; SD: standard deviation; $\mathrm{mm}^{2}$ : square millimeter; $\mathrm{mm}$ : millimeter; FAZ: Foveal Avascular Zone; Cl: Circularity Index. P<0.05 in bold. 
Table 7. Correlation Between RNFL Thickness and FAZ Descriptors or VD Defined by Univariate Regression (Intensity of Color Indicates Strength of Correlation, Expressed With r value; Blue for Positive, Red for Negative; Only Statistically Significant Marked)

\begin{tabular}{|c|c|c|c|c|c|c|c|c|c|c|c|c|c|c|c|}
\hline 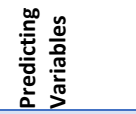 & 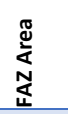 & 旁 & $\begin{array}{l}\vec{X} \\
\sum_{N} \\
\frac{N}{L}\end{array}$ & $\begin{array}{l}\overrightarrow{\mathbb{X}} \\
\sum_{N} \\
\mathbb{U} \\
\end{array}$ & 준 & 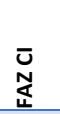 & 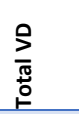 & $\begin{array}{l}0 \\
\frac{5}{5} \\
\frac{5}{2}\end{array}$ & 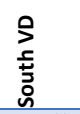 & 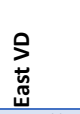 & $\begin{array}{l}9 \\
\bar{y} \\
3\end{array}$ & 3 & $\frac{0}{2}$ & $\sum_{0}^{0}$ & 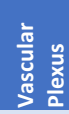 \\
\hline \multirow{2}{*}{$\begin{array}{l}\text { RNFLT } \\
\text { Correlation }\end{array}$} & -0.24 & $-0.24^{*}$ & -0.15 & -0.22 & -0.24 & 0.23 & $0.75^{* * *}$ & -0.07 & $0.51^{* *}$ & $0.49^{* *}$ & $0.62^{* * *}$ & $0.59^{* * *}$ & $0.51^{*}$ & $0.44^{*}$ & SVP \\
\hline & -0.14 & -0.12 & -0.09 & -0.11 & -0.13 & 0.18 & $0.67^{* * *}$ & 0.09 & $0.43^{*}$ & $0.69^{* * *}$ & $0.56^{* * *}$ & $0.49^{* *}$ & $0.44^{* *}$ & $0.65^{* * *}$ & DVP \\
\hline
\end{tabular}

Abbreviations: DVP: Deep Vascular Plexus; SVP: Superficial Vascular Plexus; MAL: Major Axis length; ED: Equivalent diameter; RNFLT: Retinal Nerve Fiber Layer Thickness; FAZ: Foveal Avascular Zone; Cl: Circularity Index; VD: Vessel Density; C: Circular Sector. ${ }^{*} p<0.05,{ }^{* *} p<0.01,{ }^{* * *} p<0.001$.

Automated Assessment of Macular Vascular Area and Vascular Density

Tables 5 and 6 present the comparison of the OCT-A assessed macular VD and FAZ-related parameters. In all analyzed macular areas, SVP had higher VD than deep plexus $(p<0.001)$ except in overall value, for both the glaucoma and control groups.

In SVP and DVP, the total average macular VD ( $p<0.0001$; $\mathrm{p}<0.0001$ ) was lower in the glaucoma patients compared to the controls. Additionally, the inner circular region $(p=0.04 ; p<0.0001$ respectively for SVP and DVP), DVP in $C 2$ and $C 3$ ( $p=0.01$ and $p=0.0009$, respectively) and all quadrants except for North and SVP South had a lower VD in the glaucoma patients compared to the controls.

Analysing FAZ-related parameters, the FAZ Major Axis Length $(p=0.02)$, Area $(p=0.04)$, Equivalent Diameter $(p=0.04)$ and Perimeter $(p=0.04)$ were significantly larger in eyes with POAG than the control group for SVP. The FAZ $\mathrm{Cl}$ was not different between the groups ( $p=0.95)$.

Representative cases are revealed in Fig. 2 and Fig. 3. Fig. 2 illustrate the macular vasculature degeneration associated with glaucoma and advanced glaucoma. Fig. 3 demonstrate the changes of FAZ descriptors between the members of the healthy control group, patients with glaucoma and advanced glaucoma.

\section{Structural and Functional Associations of The Macular VD and FAZ-Related Parameters}

A significant positive Spearman's rank correlation was found between all but CI SVP FAZ parameters and the MD in perimetry $(p<0.05)$. The RNFLT nasal quadrants were negatively correlated with FAZ descriptors $(p<0.05)$. The Total and Quadrant or Circular Segmented VD (from both SVP and DVP) were positively correlated with CVFS $(p<0.05)$ and the Average or Quadrant RNFLT $(p<0.05)$.
The Macular VD and FAZ-Related Parameters Predicting the Glaucoma Outcome

In the univariate regression analysis, it was found that FAZ Major axial length, Total VD and all except for North VD sectors were significantly associated with the average RNFLT $(p<0.05)$. Additionally, local irregularities of the FAZ were significantly associated with the local RNFLT (in the appropriately projected areas; $p<0.05$ ). In the regions with a decreased RNFL thickness, sharpened edges of FAZ occurred. Table 7. Multivariate linear regression models for the average RNFLT were separately built for the SVP and DVP. Interestingly, in the SVP, only the Total VD was an independent factor predicting the RNFLT (Table 8 and Fig.4 a). Model built for the SVP did not predict lower values of the RNFLT. This suggested one of the three possibilities; 1) There is an additional factor that should be incorporated; 2) The RNFLT did not depend solely on the Total VD or 3) The association is non-linear (it may be a logarithmic function). All other parameters, previously tested within the correlation analysis and associated with the RNFLT, were not considered "a stand-alone factor" in this evaluation. Differently, in the DVP, the C3 segment and East Quadrant VD both significantly contributed to predicting the RNFLT (Fig.4 b and Table 8).

The logit model probability based on the SVP showed that only the total VD was significantly associated with the probability of having glaucoma (6.78 Wald test, $p=0.009)$, Table 9. Any other parameter did not add any important (statistically significant) change in the overall model fit and therefore were withdrawn. The SVP model was analyzed with ROC ( $A \cup C=0.98, p<0.05)$, Fig.4 c). In the DVP, the C3 segment VD and FAZ area, both significantly contributed to prediction of having glaucoma (7.39 and 4.72 Wald test. $p=0.007$ and 0.03 respectively), Table 9. The DVP model was analyzed with ROC (AUC $=0.92$, $\mathrm{p}<0.05)$, Fig. 4 d. 


\section{Superficial Plexus}
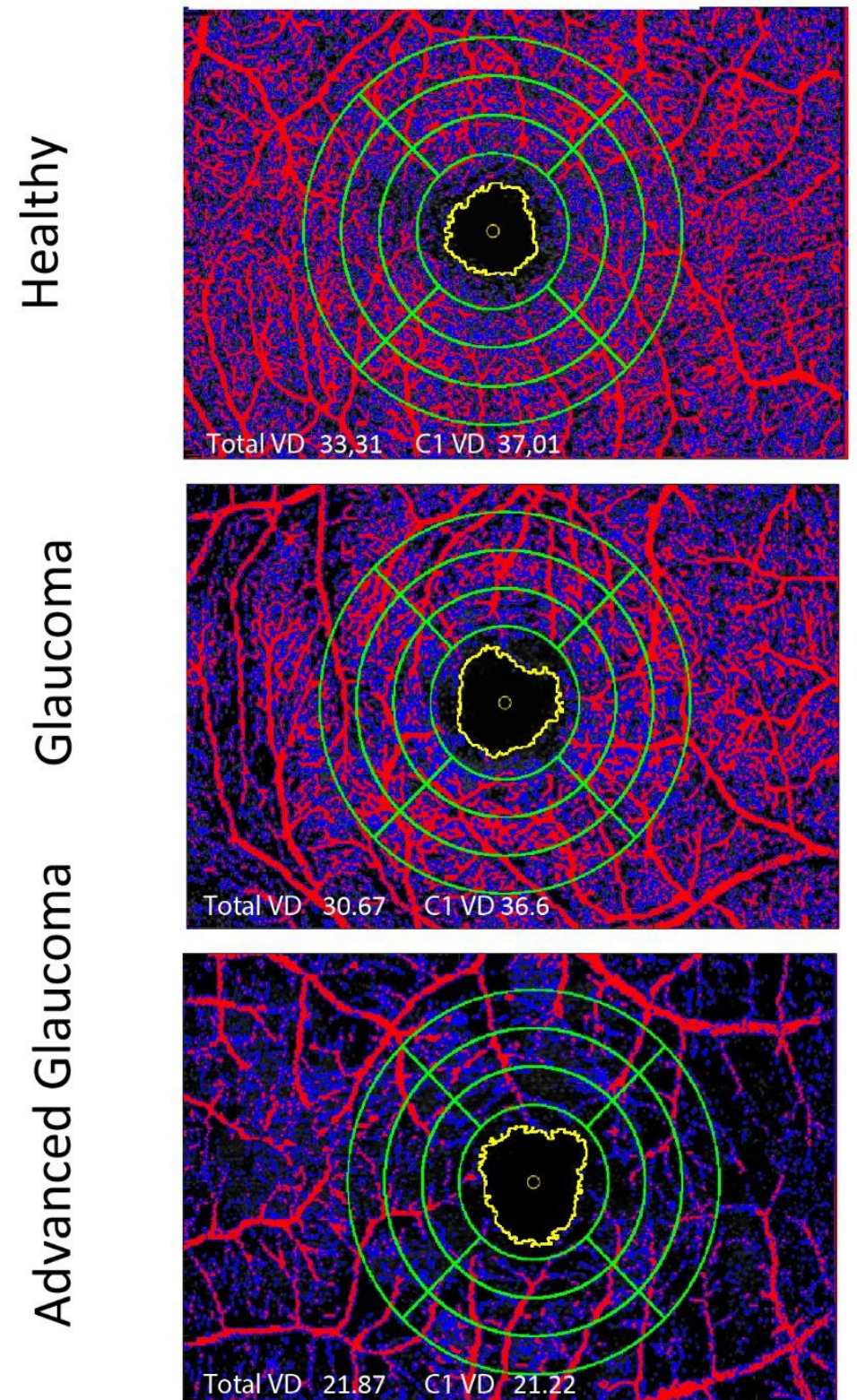

\section{Deep Plexus}
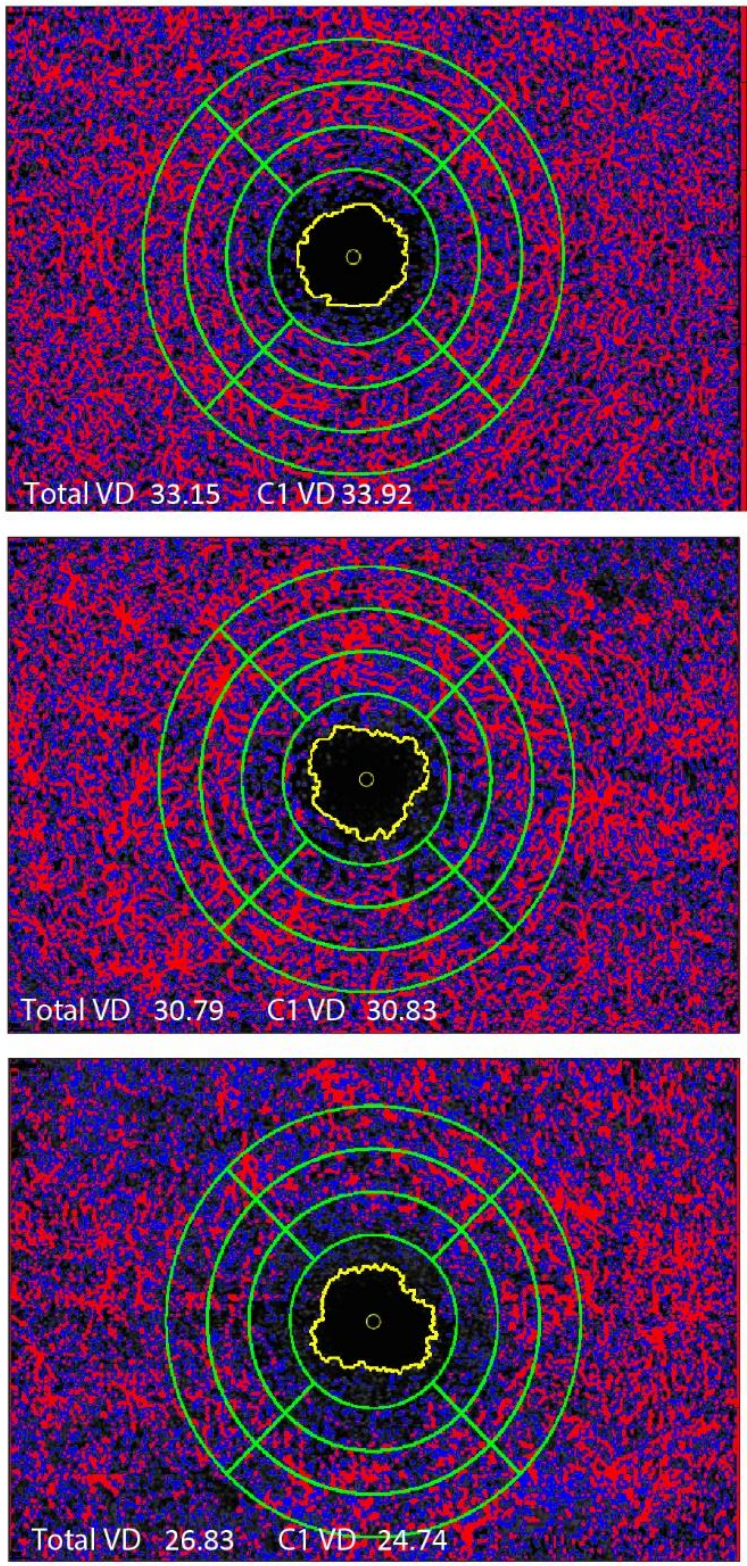

Figure 2. Representative Cases of Macular Vessel Density (VD) Assessment in Healthy, Glaucoma and Advanced Glaucoma Cases. A Significant Lower Total VD Was Found in Glaucoma Eyes, More Pronounced in Advanced Ones. Abbreviations: Total VD: Total Vessel Density; C1 VD: Inner circular area vessel density. 


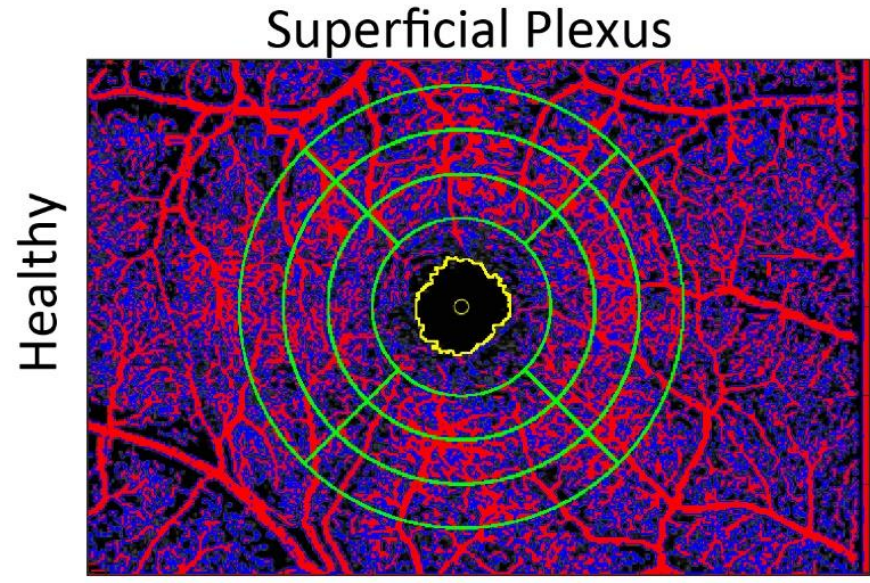

FAZ Major Axis Length 0.53 FAZ Perimeter $1.68 \mathrm{Cl} 0.91$ FAZ Area 0.23 FAZ Equivalent Diameter 0.51

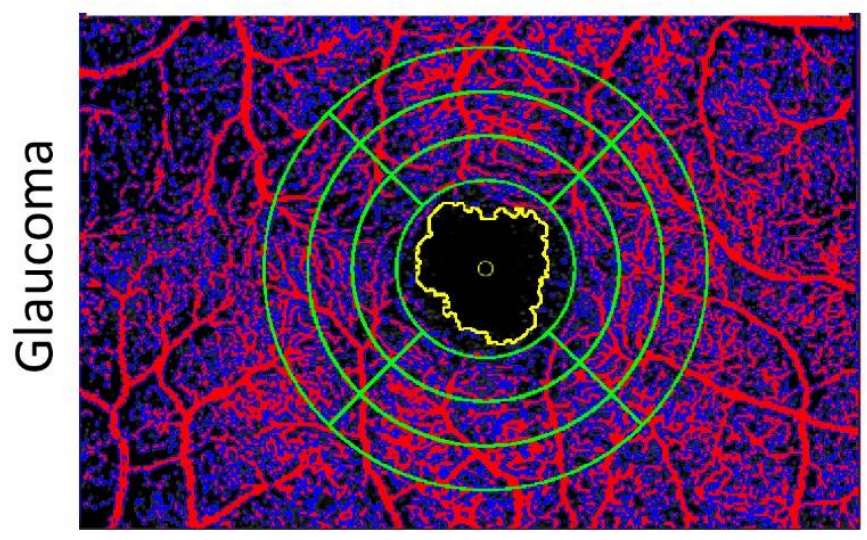

FAZ Major Axis Length 0.81 FAZ Perimeter $2.63 \mathrm{Cl} 0.78$ FAZ Area $0.43 \quad$ FAZ Equivalent Diameter 0.74

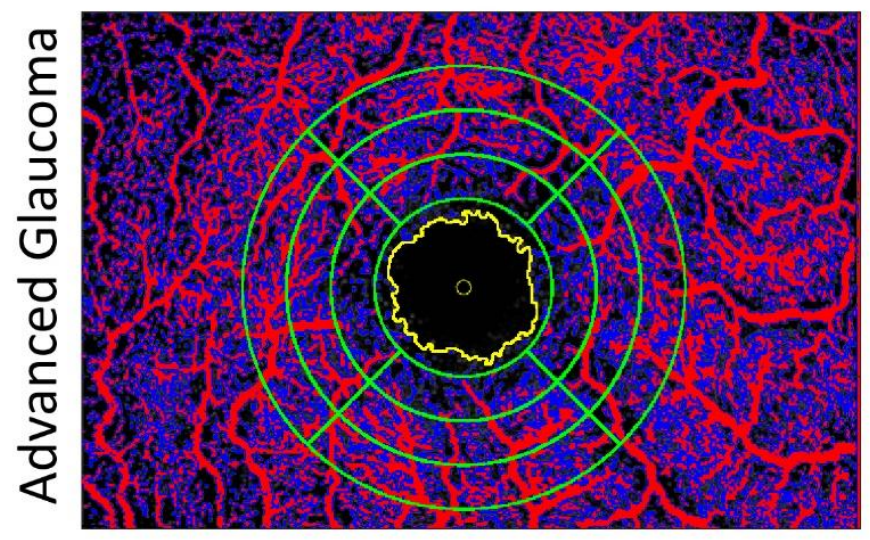

FAZ Major Axis Length 0.85 FAZ Perimeter $2.78 \mathrm{Cl} 0.84$ FAZ Area 0.52 FAZ Equivalent Diameter 0.81

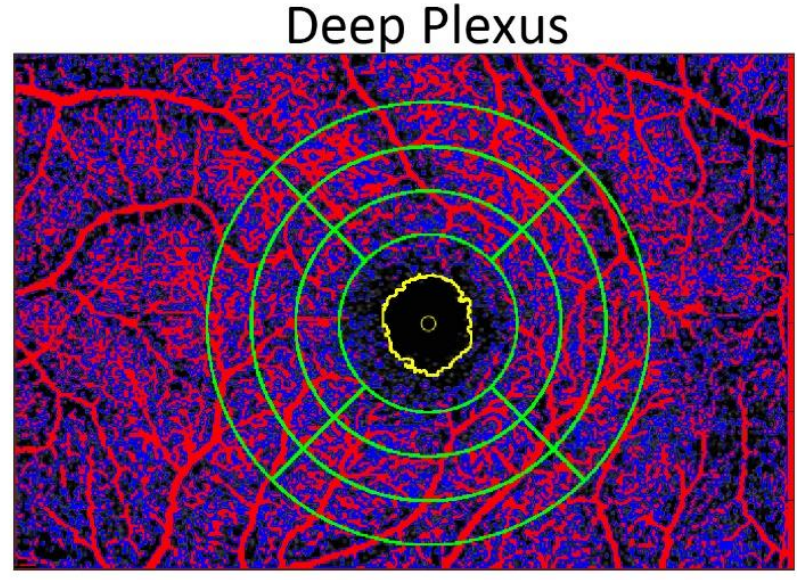

FAZ Major Axis Length 0.54 FAZ Perimeter $1.64 \mathrm{Cl} 0.97$ FAZ Area 0.20 FAZ Equivalent Diameter 0.51

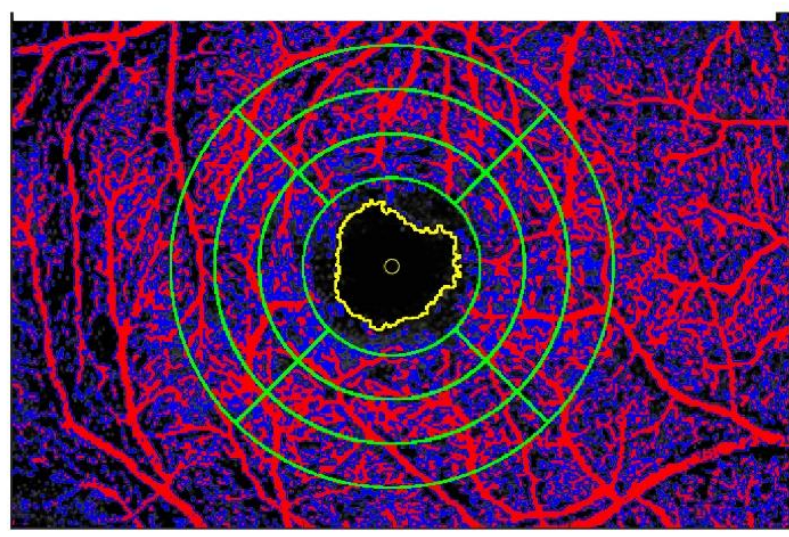

FAZ Major Axis Length 0.70 FAZ Perimeter $2.29 \mathrm{Cl} 0.85$ FAZ Area 0.36 FAZ Equivalent Diameter 0.68

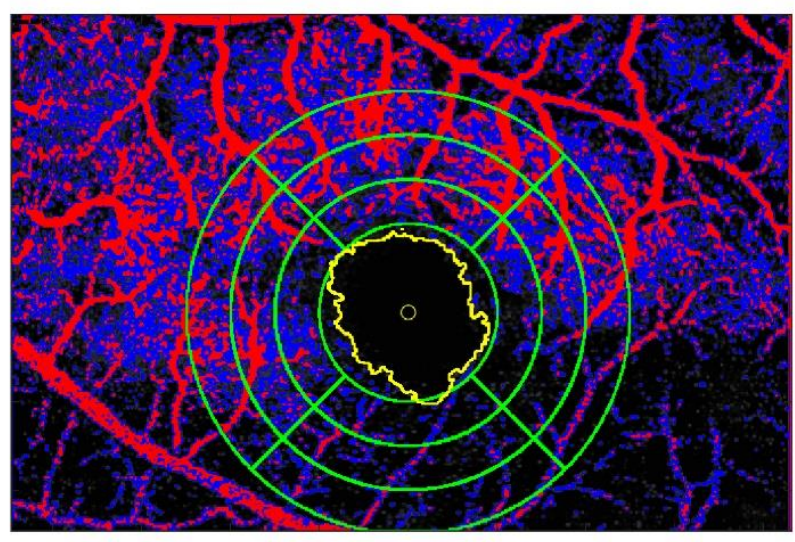

FAZ Major Axis Length 0.98 FAZ Perimeter $2.98 \quad \mathrm{Cl} 0.81$ FAZ Area 0.57 FAZ Equivalent Diameter 0.86

Figure 3. Representative Cases of Foveal Avascular Zone (FAZ) Assessment in Healthy, Glaucoma and Advanced Glaucoma Cases. FAZ Major Axis Length $(\mathrm{mm})$ and FAZ Perimeter $(\mathrm{mm})$ Were Significantly Larger in Glaucoma Group. Abbreviations: Cl: Circularity Index; mm: milimiter. 
Table 8. Significance of Different Models in Predicting RNFLT

\begin{tabular}{|l|l|l|}
\hline Model & Predicting Variable & Outcome Peripapillary RNFLT \\
\hline Based on SVP & Total VD ${ }^{* *}$ & $R^{2}=0.56 ; \beta=0.75 \pm 0.11$ \\
\hline Based on DVP & C3 Segment and East Quadrant VD & $R^{2}=0.52 ; \beta_{C 3}=0.46 \pm 0.17 ; \beta_{\text {East }}=0.33 \pm 0.17$ \\
\hline
\end{tabular}

Abbreviations: DVP: Deep Vascular Plexus; SVP: Superficial Vascular Plexus; VD: Vessel Density; RNFLT: Retinal Nerve Fiber Layer Thickness; C: Circular Sector; $\mathbf{R}^{2}$ : the R-squared. C3: Circular sector 3. ${ }^{*} p<0.05,{ }^{* *} p<0.01,{ }^{* * *} p<0.001 . \beta$ is the coefficient estimate for the independent variable that is Total VD in the SVP (value $\pm S D$ ), $\beta_{C 3}$ is the coefficient estimate for the independent variable that is C3 Segment in the DVP (value $\pm S D$ ), $\beta_{\text {East }}$ is the coefficient estimate for the independent variable that is East Quadrant VD in the DVP (value \pm SD).

Table 9. Significance of Different Models for Predicting Glaucoma Outcome.

\begin{tabular}{|l|l|l|l|}
\hline Model & Predicting Variable & Glaucoma outcome & AUC \\
\hline Based on SVP & Total VD & 6.78 Wald test, $\mathbf{p}=\mathbf{0 . 0 0 9}$ & 0.98 \\
\hline Based on DVP & C3 Segment VD & 7.39 Wald test, $\mathbf{p}=\mathbf{0 . 0 0 7}$ & 0.92 \\
& and FAZ area & 4.72 Wald test, $\mathbf{p}=\mathbf{0 . 0 3}$ & \\
\hline
\end{tabular}

Abbreviations: DVP: Deep Vascular Plexus; SVP: Superficial Vascular Plexus; VD: Vessel Density; FAZ: Foveal Avascular Zone; AUC: Area Under Curve; C: Circular Sector; VD: Vessel Density; Circular sector 3. P<0.05 in bold.
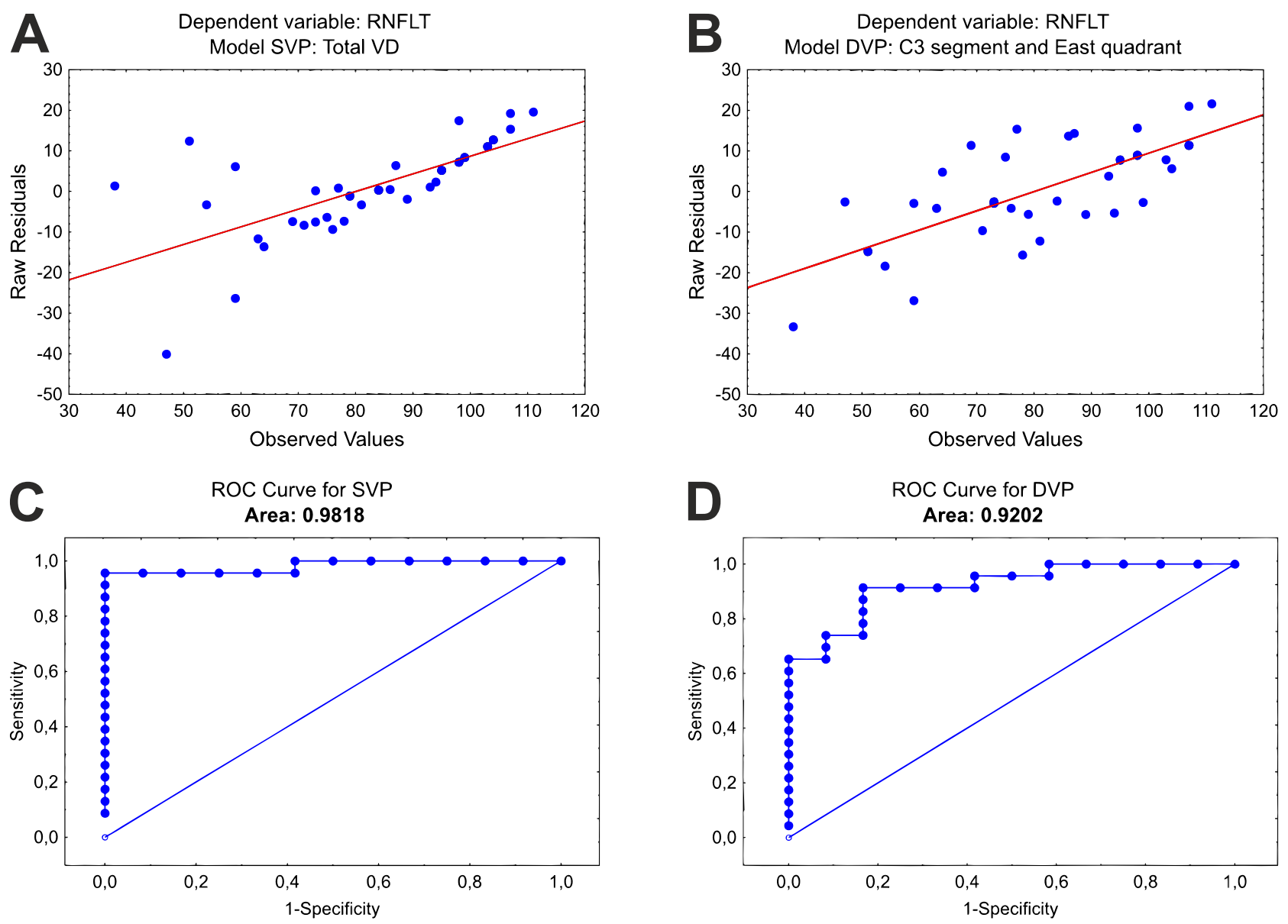

Figure 4. a) Multivariate Linear Regression Models for Average Retinal Nerve Fiber Layer Thickness (RNFLT) Built for Superficial Vessel Plexus (SVP) b) Multivariate Linear Regression Models for Average RNFLT Built for Deep Vessel Plexus (DVP). c) Receiver Operating Characteristic (ROC) Curves Analysis, SVP-Based Model d) ROC Curves Analysis, DVP-Based Model; Circular sector 3 
Table 10. The Current Pieces of Evidence on OCT-A Macular VD and FAZ Parameters for Glaucoma Assessment.

\begin{tabular}{|c|c|c|}
\hline Investigators & $\begin{array}{l}\text { Publication } \\
\text { Year }\end{array}$ & Major Findings \\
\hline Rao HL, et al. [15] & 2016 & $\begin{array}{l}\text { VD parameters had a moderate diagnostic ability to differentiate } 64 \text { POAG eyes from } 78 \text { normal eyes. } \\
\text { Peripapillary VD had significantly better diagnostic ability than ONH and macular VD. The diagnostic ability } \\
\text { improved with increasing severity. }\end{array}$ \\
\hline Xu H, et al. [15] & 2016 & $\begin{array}{l}\text { Macular capillary density was lower in } 68 \text { POAG eyes compared to } 31 \text { normal eyes. Macular capillary } \\
\text { density correlated with inner retinal thickness and VF damage. }\end{array}$ \\
\hline $\begin{array}{l}\text { Yarmohammadi A, et al. } \\
\text { [15] }\end{array}$ & 2016 & $\begin{array}{l}\text { Compared with } 28 \text { healthy eyes, reduced peripapillary and macular VD was detected in the perimetrically } \\
\text { intact hemiretina of } 58 \text { glaucoma eyes with single-hemifield defect. VD attenuation was associated with } \\
\text { the magnitude of VF changes in the corresponding hemifields. }\end{array}$ \\
\hline Chen H, et al. [15] & 2017 & $\begin{array}{l}\text { Macular and peripapillary VD was Lower in } 26 \text { POAG eyes than } 27 \text { healthy ones. The macular superficial } \\
\text { VD measurements had similar diagnostic accuracy to peripapillary RNFLT and macular Retinal Ganglion } \\
\text { Cell Layer Thickness for differentiating glaucomatous and healthy eyes. }\end{array}$ \\
\hline Kwon J, et al. [15] & 2017 & $\begin{array}{l}\text { FAZ CI was associated with central VF loss in } 78 \text { OAG patients and the FAZ area was correlated with the } \\
\text { severity of central field loss. There was a spatial correlation between the hemimacula showing enlarged } \\
\text { FAZ and the hemifield with field loss. }\end{array}$ \\
\hline Choi J, et al. [15] & 2017 & $\begin{array}{l}\text { Decreased macular VD, increased FAZ perimeter, and decreased FAZ Cl were observed in } 52 \text { POAG eyes, } \\
\text { compared to } 52 \text { healthy eyes. The later parameter was significantly correlated with a decrease in } \\
\text { peripapillary thickness of the RNFL and showed the highest area under receiver operating characteristics. }\end{array}$ \\
\hline
\end{tabular}

Abbreviations: OCTA: Optical Coherence Tomography Angiography; VD: Vessel Density; FAZ: Foveal Avascular Zone; POAG: Primary Open Angle Glaucoma; ONH: Optic Nerve Head; VF: Visual Field; RNFL: Retinal Nerve Fiber Layer; RGC: Retinal Ganglion Cell; Cl: Circularity Index; OAG: Open Angle Glaucoma.

RGC death

\section{Glaucoma}

Impaired vascular autoregulation Abnormal neurovascular coupling Oxidative Stress and Ishemia

Elevated IOP Vascular Compression

\section{Reduced OPP}

Reduced blood flow of ONH and Macula

Vascular Dropout

Vessel Density reduction

Irregular FAZ outline

FAZ enlargement

Figurer 5. POAG Vascular Risk Schematic Diagram.

Abbreviations: POAG: Primary Open Angle Glaucoma; RGC: Retinal Ganglion Cell; IOP: Intraocular Pressure; OPP: Ocular Perfusion Pressure; ONH: Optic Nerve Head; FAZ: Foveal Avascular Zone.

\section{DISCUSSION}

In the current study, the macular VD and FAZ biometry were quantitatively demonstrated in POAG using OCT-A.
The results confirmed the previously described association between a decreased macular VD and glaucoma development $[15,19,20]$. It also showed the association of FAZ-related parameters with structural and functional glaucoma outcomes. The macular VD had a diagnostic value discriminating patients with glaucoma and healthy ones.

Differently from previous report by Choi et al., the FAZ was assessed automatically, simultaneously to the vascular density. Firstly, this enables us to reduce the source of bias from two different methods of assessment. As the evaluation of the FAZ in the ImageJ separately from VD increases the possible error. Secondly, in this study the large and small macular vessels density were assessed separately but again during one image processing protocol. The operator did not have any influence on the image processing protocol. This again differs from what was presented by Choi et al [15].

$\mathrm{ONH}$, retina and choroid circulation defects were suspected to be involved in glaucoma pathogenesis [21]. It is already accepted that Ocular blood flow (OBF) has an important role in Normal Tension Glaucoma (NTG). Additionally, a study with 51 patients with chronic POAG found a retinal vasculature circulatory deficit. Mean dye velocity reduction and arteriovenous passage time prolongation were found in fluorescein angiograms [22, 23]. Table 10 summarizes the current pieces of evidence on the glaucoma application of OCT-A in the assessment of the macular VD and FAZ biometrics [15, 19, 24-27]. The macula is of great clinical importance to glaucoma. Anatomically, it accommodates $50 \%$ of RGCs [15]. Physiologically, the retina demands more oxygen than 
other metabolically active tissues, as the brain [28]. Changes of vascular networks in neurodegenerative and neuroischemic defects drive to hypoxic/anoxic events or hypoxia-reperfusion injuries and biochemical changes, which ultimately compromise cellular function [29]. The macular susceptibility to hypoxic/ischemic damage as well as reperfusion injury was previously described. To maintain an appropriate sensory transmission, it requires a functional vasculature network with high rates of perfusion [29]. Deficient oxygen supply and tissue hypoxia are involved in RGC death, resulting in loss of vision [16, 29].

In the final pathway, hypoxia causes activation of microglial cells with consequent release of TNF- $\alpha$ and IL$1 \beta$. The hypoxic retinal tissue also releases glutamate in excess that activates ionotropic receptors, leading to excitotoxicity by an increased RGC's calcium influx. Both of these pathways result in RGC death through caspase signaling, mitochondrial dysfunction and oxidative stress [28].

POAG vascular changes may be the primary etiology or the consequence of glaucoma mechanisms (Figure 5). On the one hand, blood flow impaired autoregulation may be caused by glaucoma oxidative stress-induced vascular alterations [1]. Moreover, hypertension mechanisms may induce vascular compression and breakdown. The absence of pathologic neovascularization associated with the ischemic retina supports microvascular degeneration as a primary mechanism.

The recent OCT-A technology allowed specific macular retinal layer VD assessment. Hemodynamic quantification performed by means of OCT-optical microangiography and tissue perfusion images have shown correlation with blood flow in vivo [30].

Rao et al. [19] reported that the macular VD, evaluated by OCT-A, was decreased in a glaucoma group compared to healthy individuals. In our study, loss of capillaries in POAG was reflected in the sectorial macular VD analysis for both superficial and deep vessel plexus.

In the previous publications, the FAZ morphology has been indicated as a diagnostic and prognostic parameter for vascular retinal diseases [27, 28]. FAZ descriptors are relatively unused parameters in the field of glaucoma. Previous reports demonstrated that decreased FAZ circularity and an increased FAZ area were significantly associated with the presence and severity of CVFD at onset [27]. In this study, we found that enlargement of FAZ major axis length, area, equivalent diameter and perimeter were observed in the glaucoma group compared to the controls. Loss of the parafoveal capillary network changes FAZ biometry in glaucoma. These parameters may be associated with the status of macular vascular perfusion and disintegration of vascular arcades. In this regard, the FAZ parameters appeared to be a significant disease indicator due to their pathomorphological status. We must consider changes of FAZ morphology and disintegration of vascular arcades as a novel path into understanding the glaucoma pathophysiology.

In contrast to a previous report [15], FAZ - Cl was not among the best discriminating parameters between eyes with glaucoma and healthy ones. Even if a subtle change of the parafoveal vascular arcade and a more irregular FAZ outline were found, FAZ enlargement was statistically more significant than $\mathrm{Cl}$. In this study, unlike the previous one, the FAZ parameters were automatically driven from the image processing and no potential human error was induced.

A previous report [26] found that POAG eyes had a diminished macular VD. This result was highly associated with severe macular and perimacular VF defects as well as a thinner retinal thickness in corresponding areas. Similarly, in this work, an association was found between the macular VD and CVF sensitivity along with a positive correlation between FAZ descriptors (except for $\mathrm{Cl}$ ) and the MD. These findings showed that a decreased microvasculature structure may be correlated with functional glaucomatous damage.

SVP Total macular VD and DVP Segment C3 VD and FAZ area performed well in terms of the logit modeled probability of having glaucoma. Multiple regression disclosed a consistent association between SVP Total VD and the DVP's both East and C3 sectors with the peripapillary RNFLT. Those findings, similar to the previous work of Choi et al., suggest that disruption of parafoveal vascular blood supply is related to structural glaucomatous damage and then has a diagnostic value [15].

This report emphasizes the vascular risk of POAG pathogenesis and its accurate quantitative assessment. Combining the macular VD and FAZ biometry with RNFLT structural damage can be useful. It may anticipate glaucoma diagnosis, thus preventing early damage.

To know whether the vascular or the hypertensive powered mechanism comes first, a better understanding of how the neurovascular unit reacts under both stress factors is required. It is desirable to recognize the microvascular arterial and venous differential impact and to evaluate the relationship between microcirculatory temporal changes in different glaucoma stages. Future studies are needed to appreciate the importance of these biometric parameters in different types of glaucoma (e.g. 
NTG). The retrospective design, small sample size, no agematching groups and no adjustment of refractive error or glaucoma severity were some limitations. Also, there may be unrecognized confounding variables affecting macular microvascular perfusion status.

\section{CONCLUSION}

The present study evaluated different OCT-A vascular parameters such as the macular VD and FAZ morphology in glaucoma in vivo and quantitatively. A decreased macular VD, increased FAZ major axis length, area, equivalent diameter and perimeter, were found in the eyes with glaucoma compared with healthy eyes. The macular VD predicted RNFLT effectively and showed considerable value for glaucoma diagnosis. It may be a novel biomarker, representing a disruption of microvascular macular perfusion.

The current algorithm, automatically, assessed the quantitative macular capillary network. The authors demonstrated its potential application in a clinical or research setting, especially in the field of vascular pathogenesis.

This study suggested that a macular vascular dropout plays an important role in POAG neurodegeneration.

\section{ETHICAL DECLARATIONS}

Ethical Approval: This study conformed to the tenets of the Declaration of Helsinki and received an ethical approval from Portuguese National Network of Ethics Commissions, Registration Number 20170700050.

Conflict of Interest: None.

\section{FUNDING}

Publication grant aimed to cover Article Processing Fee, was funded by Wroclaw Medical University Subvention for The Department of Ophthalmology (grant no: SUB.C240.19.058). The funders had no role in study design, data collection and analysis, decision to publish, or preparation of the manuscript.

\section{ACKNOWLEDGMENT}

None.

\section{REFERENCES}

1. Chrysostomou V, Rezania F, Trounce IA, Crowston JG. Oxidative stress and mitochondrial dysfunction in glaucoma. Curr Opin Pharmacol. 2013;13(1):12-5. doi: 10.1016/j.coph.2012.09.008 pmid: 23069478

2. Wang $\mathrm{H}$, Wang R, Thrimawithana T, Little PJ, Xu J, Feng $\mathrm{ZP}$, et al. The nerve growth factor signaling and its potential as therapeutic target for glaucoma. Biomed Res Int. 2014;2014:759473. doi: 10.1155/2014/759473 pmid: 25250333 3. Susanna R, Jr., Clement C, Goldberg I, Hatanaka M. Applications of the water drinking test in glaucoma management. Clin Exp Ophthalmol. 2017;45(6):625-31. doi: 10.1111/ceo.12925 pmid: 28164419

4. Poon YC, Teng MC, Lin PW, Tsai JC, Lai IC. Intraocular pressure fluctuation after water drinking test in primary angleclosure glaucoma and primary open-angle glaucoma. Indian J Ophthalmol. 2016;64(12):919-23. doi: 10.4103/03014738.198851 pmid: 28112134

5. Susanna R, Jr., Vessani RM, Sakata L, Zacarias LC, Hatanaka $M$. The relation between intraocular pressure peak in the water drinking test and visual field progression in glaucoma. $\mathrm{Br} J$ Ophthalmol. 2005;89(10):1298-301. doi: 10.1136/bjo.2005.070649 pmid: 16170120

6. Akagi T, lida Y, Nakanishi H, Terada N, Morooka S, Yamada $\mathrm{H}$, et al. Microvascular Density in Glaucomatous Eyes With Hemifield Visual Field Defects: An Optical Coherence Tomography Angiography Study. Am J Ophthalmol. 2016;168:237-49. doi: 10.1016/j.ajo.2016.06.009 pmid: 27296492

7. Shahlaee A, Pefkianaki M, Hsu J, Ho AC. Measurement of Foveal Avascular Zone Dimensions and its Reliability in Healthy Eyes Using Optical Coherence Tomography Angiography. Am J Ophthalmol. 2016;161:50-5 e1. doi: 10.1016/j.ajo.2015.09.026 pmid: 26423672

8. Rosenfeld PJ, Durbin MK, Roisman L, Zheng F, Miller A, Robbins G, et al. ZEISS Angioplex Spectral Domain Optical Coherence Tomography Angiography: Technical Aspects. Dev Ophthalmol. 2016;56:18-29. doi: 10.1159/000442773 pmid: 27023249

9. Gołębiewska J, Hautz W. Angio-OCT in ophthalmological diagnostics and therapy - part I. Ophtha Therapy. 2016;3(3):161-71.

10. Bojikian KD, Chen CL, Wen JC, Zhang Q, Xin C, Gupta D, et al. Optic Disc Perfusion in Primary Open Angle and Normal Tension Glaucoma Eyes Using Optical Coherence TomographyBased Microangiography. PLoS One. 2016;11(5):e0154691. doi: 10.1371/journal.pone.0154691 pmid: 27149261

11. Wang $X$, Jiang $C$, Ko T, Kong $X, Y u X$, Min W, et al. Correlation between optic disc perfusion and glaucomatous severity in patients with open-angle glaucoma: an optical coherence tomography angiography study. Graefes Arch Clin Exp Ophthalmol. 2015;253(9):1557-64. doi: 10.1007/s00417-0153095-y pmid: 26255817

12. Leveque PM, Zeboulon P, Brasnu E, Baudouin C, Labbe A. Optic Disc Vascularization in Glaucoma: Value of SpectralDomain Optical Coherence Tomography Angiography. J 
Ophthalmol. 2016;2016:6956717. doi: 10.1155/2016/6956717 pmid: 26998352

13. Yarmohammadi A, Zangwill LM, Diniz-Filho A, Suh MH, Manalastas PI, Fatehee N, et al. Optical Coherence Tomography Angiography Vessel Density in Healthy, Glaucoma Suspect, and Glaucoma Eyes. Invest Ophthalmol Vis Sci. 2016;57(9):OCT4519. doi: 10.1167/iovs.15-18944 pmid: 27409505

14. Riva C, Alm A, Pournaras C. Ocular circulation. In: Levin L, Nilsson S, Ver Hoeve J, Samuel M, editors. Adler's Physiology of the Eye. 11th ed. Philadelphia, USA: Saunders Elsevier; 2011. 15. Choi J, Kwon J, Shin JW, Lee J, Lee S, Kook MS. Quantitative optical coherence tomography angiography of macular vascular structure and foveal avascular zone in glaucoma. PLoS One. 2017;12(9):e0184948. doi: 10.1371/journal.pone.0184948 pmid: 28934255

16. Shaffer R. A New Classification of the Glaucomas. Trans Am Ophthalmol Soc 1960;58:219-25.

17. Pizer SM, Amburn EP, Austin JD, Cromartie R, Geselowitz A, Greer T, et al. Adaptive histogram equalization and its variations. Computer Vision, Graphics, and Image Processing. 1987;39(3):355-68. doi: 10.1016/s0734-189x(87)80186-x

18. Yousefi S, Qin J, Zhi Z, Wang R. Uniform enhancement of optical micro-angiography images using Rayleigh contrastlimited adaptive histogram equalization. Quant Imaging Med Surg 2013;3:5-17. doi: 10.3978/j.issn.2223-4292.2013.01.01

19. Rao HL, Pradhan ZS, Weinreb RN, Reddy HB, Riyazuddin M, Dasari S, et al. Regional Comparisons of Optical Coherence Tomography Angiography Vessel Density in Primary Open-Angle Glaucoma. Am J Ophthalmol. 2016;171:75-83. doi: 10.1016/j.ajo.2016.08.030 pmid: 27590118

20. Spraul CW, Lang GE, Lang GK, Grossniklaus HE. Morphometric changes of the choriocapillaris and the choroidal vasculature in eyes with advanced glaucomatous changes. Vision Research. 2002;42(7):923-32. doi: 10.1016/s00426989(02)00022-6

21. Nicolela MT, Hnik P, Drance SM. Scanning Laser Doppler Flowmeter Study of Retinal and Optic Disk Blood Flow in Glaucomatous Patients. American J Ophthalmology. 1996;122(6):775-83. doi: 10.1016/s0002-9394(14)70373-3

22. Wolf S, Arend O, Sponsel WE, Schulte K, Cantor LB, Reim M. Retinal Hemodynamics Using Scanning Laser Ophthalmoscopy and Hemorheology in Chronic Open-angle
Glaucoma. Ophthalmology. 1993;100(10):1561-6. doi: 10.1016/s0161-6420(93)31444-2

23. Fuchsjager-Mayrl G, Wally B, Georgopoulos M, Rainer G, Kircher K, Buehl W, et al. Ocular blood flow and systemic blood pressure in patients with primary open-angle glaucoma and ocular hypertension. Invest Ophthalmol Vis Sci. 2004;45(3):8349. doi: 10.1167/iovs.03-0461 pmid: 14985298

24. Yarmohammadi A, Zangwill LM, Diniz-Filho A, Saunders LJ, Suh $\mathrm{MH}, \mathrm{Wu} Z$, et al. Peripapillary and Macular Vessel Density in Patients with Glaucoma and Single-Hemifield Visual Field Defect. Ophthalmology. 2017;124(5):709-19. doi: 10.1016/j.ophtha.2017.01.004 pmid: 28196732

25. Chen HS, Liu CH, Wu WC, Tseng HJ, Lee YS. Optical Coherence Tomography Angiography of the Superficial Microvasculature in the Macular and Peripapillary Areas in Glaucomatous and Healthy Eyes. Invest Ophthalmol Vis Sci. 2017;58(9):3637-45. doi: 10.1167/iovs.17-21846 pmid: 28728171

26. $\mathrm{Xu} \mathrm{H}, \mathrm{Yu} J$, Kong $X$, Sun $X$, Jiang $C$. Macular microvasculature alterations in patients with primary openangle glaucoma: A cross-sectional study. Medicine (Baltimore). 2016;95(33):e4341. doi: 10.1097/MD.0000000000004341 pmid: 27537559

27. Kwon J, Choi J, Shin JW, Lee J, Kook MS. Alterations of the Foveal Avascular Zone Measured by Optical Coherence Tomography Angiography in Glaucoma Patients With Central Visual Field Defects. Invest Ophthalmol Vis Sci. 2017;58(3):163745. doi: 10.1167/iovs.16-21079 pmid: 28297029

28. Kaur C, Foulds WS, Ling EA. Hypoxia-ischemia and retinal ganglion cell damage. Clin Ophthalmol. 2008;2(4):87989. doi: 10.2147/opth.s3361 pmid: 19668442

29. Sapieha P. Eyeing central neurons in vascular growth and reparative angiogenesis. Blood. 2012;120(11):2182-94. doi: 10.1182/blood-2012-04396846 pmid: 22705597

30. Yousefi S, Qin J, Wang RK. Super-resolution spectral estimation of optical micro-angiography for quantifying blood flow within microcirculatory tissue beds in vivo. Biomed Opt Express. 2013;4(7):1214-28. doi: 10.1364/BOE.4.001214 pmid: 23847744 